\title{
Driver Intention Recognition Method Using Continuous Hidden Markov Model
}

\author{
Haijing Hou, Lisheng Jin*, Qingning Niu, Yuqin Sun \\ Transportation College, Nanling Campus of Jilin University, Changchun, Jilin, China \\ Meng Lu \\ Royal Dutch Society of Engineers (KIVI NIRIA), Netherlands
}

Received: 10-03-2011

Accepted: 19-04-2011

\begin{abstract}
In order to make Intelligent Transportation System (ITS) work effectively, a driver intention recognition method is proposed. In this research, three different recognition models were developed based on Continuous Hidden Markov Model (CHMM), and could distinguish left and right lane change intention from normal lane keeping intention. Subjects performed lane change maneuvers and lane keeping maneuvers with driving simulator which simulated highway scenes, parameters that highly correlated with lane change behavior were collected and analyzed. A series of testings and comparisons were done to obtain the optimal model structure and feature set. Results show that, taking the steering wheel angel, steering wheel angle velocity and lateral acceleration as the optimal observation signals, the accuracy can achieve up 95\%, and it proved very effective in terms of early intention recognition.
\end{abstract}

Keywords: Intelligent Transportation System, driver intention recognition, CHMM, lane change

\section{Introduction}

With the increased risk of driving, vigorous efforts are under way today to develop Intelligent Transportation System (ITS) technologies, which aim at improving the performance of the whole driver-vehicle-road cooperative system ${ }^{1-2}$.With the idea of integrated deployment, safety, mobility, and efficiency, ITS has been recognized as the most effective solution for modern traffic problems. Advanced Driver Assistance Systems (ADAS) is a key part of ITS definitely, which used to provide driver with safety and comfort. However, with the growing complexity of ITS, it calls for a higher-level coordination between various ADAS sub-systems ${ }^{3}$. Therefore, the fundamental and important requirement for these systems is continuous monitoring and understanding of the current driver intention and the situation it is involved in. In various research studies, situation perception has been extensively studied from different perspectives, such as based on vision information $^{4-5}$, based on the fusion of lader and vision information $^{6-8}$. These systems aim to monitor the current traffic situation it is involved in, and assess the criticality of the situation, which in turn can be used to make informed decisions, avoid risks, and, in general, improve the performance of the system. Besides, human factors are also critical to the successful implementation of ITS $^{3,9-10}$. These assistant systems may make false alarm because of lacking of the information of the driver intention, for example, Obstacle and Collision

* Corresponding author: jinls@jlu.edu.cn 
Warning system (OCW) would work when the driver is going to change lane, and this could reduce the acceptance of ADAS. Besides, safety systems that automatically intervene in the vehicle dynamics may operate against with one another without knowing the driver's expected behavior. Accordingly, to make any such ADAS sub-system work effectively- i.e. offer the right kind of help at the right time-it is imperative to have an idea of the driver's current intention. It is not sufficient to rely exclusively on the turn signal for intention recognition, since many drivers cannot use the signals reasonably. Olsen ${ }^{11}$ showed that turn signals are only used in $64 \%$ of the lane changes, while some drivers turn on the signal after the lane change starts instead of the initial phase. Therefore, we need to explore more other characterizations of driving behavioral parameters to recognize driver intention.

Recognition of driver intention has been explored in various studies. Since driver intention recognition is basically pattern recognition and condition monitoring problem, most published systems are based on well known techniques in this area, such as Support Vector Machines $^{12}$, neural network ${ }^{13}$ and so on. Joel C.McCall et al. ${ }^{14}$ use a Bayesian framework to support a braking assistant by assessing the braking probability and the probability that the driver intends to perform a braking action. Salvucci et al. $^{15}$ proposed a mind tracking approach that uses a cognitive architecture called ACT-R to model driver's behavior. However, this system does not utilize the fact that the measurable parameters indicating the driver state may often reside in some high dimensional feature space and data can be easily classified by finding a linear separating hyperplane. Some recent endeavors in this direction mainly use a popular methodology-Hidden Markov Models (HMM) ${ }^{16}$, such as work from Pentland et al. ${ }^{17}$, Mizushima et al. ${ }^{18}$, and Meyer-Delius et al. ${ }^{19}$. It is a probabilistic model supporting recognition of temporal data patterns. These approaches were based on the concept that human behavior is made up of a sequence of internal 'mental' states that was not directly observable, while it can be inferred from observable signals, such as the steering wheel angle and velocity, yaw rate, and pedal positions.

However, the recognizers mentioned above are still far from perfect, especially for Chinese driver in China traffic situation. For example, in Pentland's ${ }^{17}$ research, they offer only discrete recognition which oppose to continuous recognition (i.e. real-time recognition). In Meyer-Delius' paper ${ }^{19}$, it only considers three situations, namely passing, aborted passing, and following, which could not generalize typical driver behavior in highway. Besides, the point is that their subjects all drive on the left side of the road.

In this research, we present a framework based on CHMM for modelling and recognizing of driver intentions. Although the framework is generic, we focus on a driver assistant application in traffic scenarios that typically occur in China highway-like driving settings, and the intentions recognized by our current system include Lane Change (left \&right), Lane Keeping. This work aims to obtain the optimal recognition models based on HMM for Chinese typical highway driving scenarios, which are especially suitable to the driving habits and behavioural norms of Chinese driver. In the following sections the system development is introduced, including the experimental platform that has been developed, and the host vehicle dynamic data collection and analysis. In addition, the recognition model training and developing process is also elaborated. Furthermore, the recognition results are presented and some conclusions are drawn.

\section{Data Acquisitions}

To collect and analyze data about the driving behavior of Chinese drivers, a PC-based interactive simulating experimental platform-Santana 2000 driving simulator has been developed, which allows the user to control almost all aspects of driving such as throttling, breaking, and steering, and includes advanced data collection and analysis modules. Figure 1 shows the experimental platform infrastructure. The driving scenario, including weather, road condition, traffic lights and other signs, pedestrian, buildings, and so on, was carefully designed by professionals to make the simulation as close to reality as possible. During driving process, the simulator outputs 398 different dynamic parameters simultaneously. Table 1 lists the six parameters used in our system. These parameters are selected because they are suggested to be highly correlated to lane change behavior and can be extracted reliably in real vehicles.

Ten driver subjects with different years of driving experience were instructed to use this simulator to drive 
through a computer graphics world. This world analogized from highway situation which contains a number of buildings, many roads with standard markings, and other moving cars. Each subject drove through the simulated world at the speed of $80 \mathrm{kph}$ to $120 \mathrm{kph}$ for approximately 40 minutes, during which time about 60 lane change maneuvers were carried out, and information about the seven parameters were recorded at 0.1 second intervals.

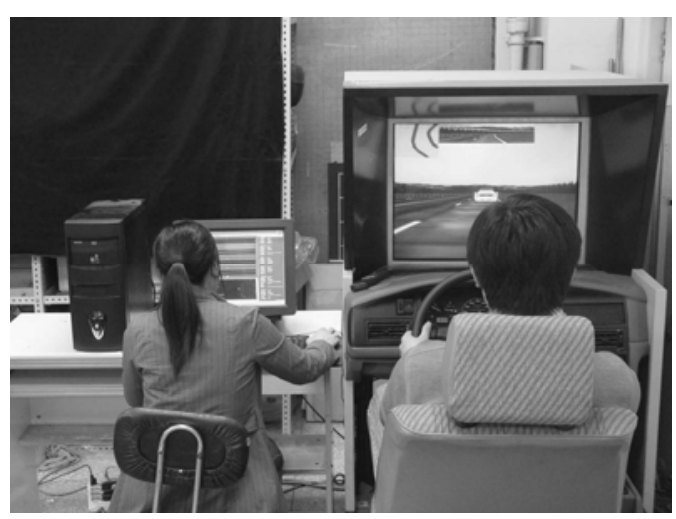

Fig. 1. Experimental platform infrastructure

The raw vehicle dynamic parameter data stream is converted into a sequence of feature vectors for modelling. They were investigated to find the correlation with the lane change manoeuvre and the change regularity during the process. Figure 2 shows the change of the mentioned parameters during LCL、

LCR 、 LK manoeuvre. In the sub-figures, $\mathrm{X}$-axis represents the time of each manoeuvre, $\mathrm{Y}$-axis stands for the parameters value according to their respective unit. It is important to note that values of parameters P1 and P5 have been narrowed ten times, aiming at showing the variation of the parameters more clearly. It can be seen that the better signal-to-noise-ratio of the steering wheel angle (P4) makes it the dominant signal in this case. Besides, steering wheel angle velocity (P3) and lateral acceleration (P6) also show great difference between lane change manoeuvre and lane keeping manoeuvre. However, Car speed (P1), Transmission Position (P2) and acceleration pedal position (P3) cannot show significant differences in different manoeuvres. In conclusion, the best recognition performance was reached with a model that was trained with sequences of steering wheel angle, steering wheel angle velocity and lateral acceleration.

Table 1. Vehicle dynamic parameters used in our system

\begin{tabular}{cc}
\hline ID & Description \\
\hline P1 & car speed(m/s) \\
\hline ID & Description \\
\hline P2 & Transmission Position \\
P3 & steering angle velocity(rad/s) \\
P4 & steering angle (rad) \\
P5 & acceleration pedal position $(\%)$ \\
P6 & lateral acceleration $\left(\mathrm{m} / \mathrm{s}^{2}\right)$ \\
\hline
\end{tabular}

\section{HMM recognition model}

\subsection{Choosing the HMM Structure}

Driver behavior is a continuous process, and the current state only relies on the last state. Based on these characterizes of lane change manoeuvre, the left-right HMMs were chosen. Figure 3 below shows a left-right HMM. In such an HMM type, the model either can stay in a particular state or transition to the next state in the forward direction. The model cannot skip states or transition to any state in the backward direction. For ADAS, it is much more interesting to identify a manoeuvre at a very early stage after it was started. This can be accomplished with continuous recognition, where a fixed-length time window of signals is batched and continuously tested against the Markov models.

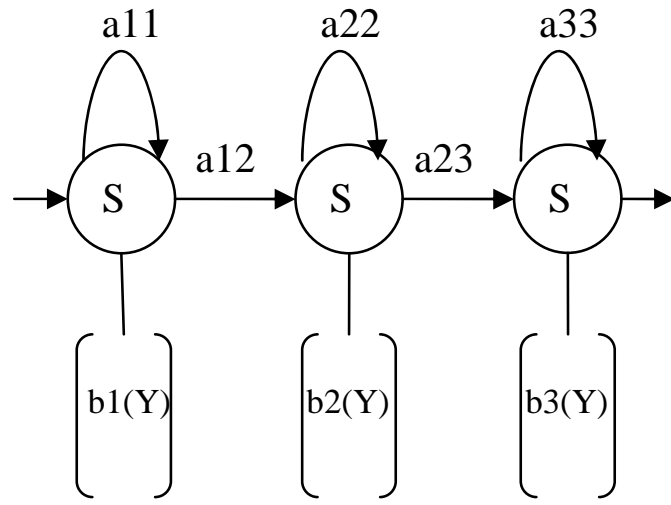

Fig. 3. Structure of left-right CHMM 
With the definition of the left-right structure and continuous recognition, the problem domain is clearer.

Given an observation sequence $O$ and the number of states and mixtures, a continuous HMM (CHMM) can be characterized by Equation (1):

$$
\lambda=[\pi, A, C, \mu, U]
$$

Here, $\pi$ is the initial state distribution ; $A$ is the state transition probability distribution; $C$ is the mixture coefficient for every probability density function (pdf) ; $\mu$ is the mean vector ; $U$ is the covariance matrix.

\subsection{Training $H M M$}

Understanding the differences between the lane change behavior and the lane keeping behavior, is a prerequisite for developing the relevant intention model. There are various kinds of definition about lane change. However the definition of lane changing used in Refs.15 was found to be most reasonable. Accordingly, a lane changing manoeuvre starts when the vehicle moves towards the other lane boundary and never returns i.e. the final shift of the driver towards the other lane. Any reversal cancels the lane change. The lane change ends when the vehicle crosses the lane boundary. Here, we divide two lane changing manoeuvre: left lane change (LCL) and right lane change (LCR).

A statistical model, such as a Hidden Markov Model, has to be trained with labeled sample data to determine the model parameters in an optimal fashion before it can be used for intention recognition ${ }^{20}$, see Figure 4.Based on the training set, the Baum-Welch algorithm can be used for parameter estimation. The implementations of the HMM standard algorithms of the Hidden Markov Toolkit (HTK) ${ }^{21-22}$ have been used in this work.

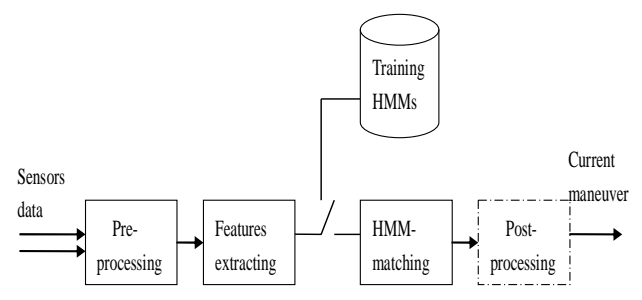

Fig. 4. CHMM training and evaluating structure
During the training process, these three intention models: LCL、 LCR and LK were training separately based on their own data base. While training the HMMs the most important factors that affected the results were data representation, window size, and initial sets of model parameters, experiments were conducted with different configurations of each of these factors to obtain the best recognition system. Parameter estimation was completed in the following stages.

(i) Create an HMM definition script, which includes the number of states and mixtures, the dimension of the data, the structure of the HMM. Here we set the left-right structure, and the number of mixtures is 3.For each model, various models with different numbers of states and sizes of time window have been trained and tested later (e.g. three states \&4s window size, six states \&2s window size etc).

(ii) Deal with the original database, and then create the separate set of training samples for LCL, LCR and LK. Median filter was used to smooth the original data. As it was expected that the steering wheel angel, the steering wheel angle velocity and lateral acceleration are the most dominant feature of the available signals, they have been taken as the optimal observation signals. For the two types of lane change situations, data was extracted between command execution and the first peak of the steering angle according to the size of time window. For lane keeping, data was extracted for the same interval with the lane change situation. See Figure 5.

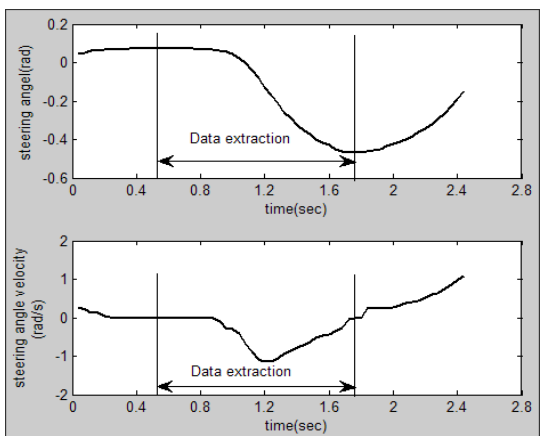

(a) a LCL maneuver 


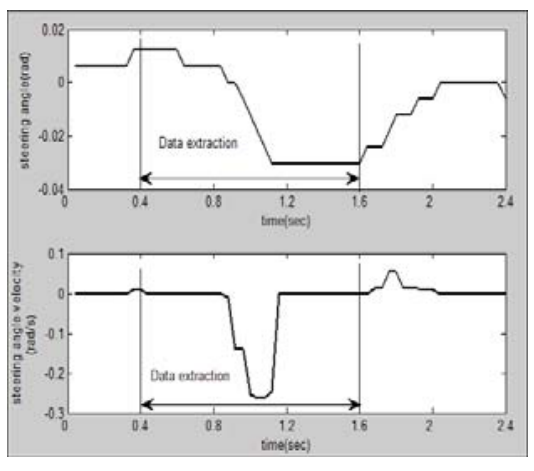

(b) a LK maneuver

Fig. 5. Data extraction method (take steering angel and steering angel velocity for example)

(iii) Choose an initial set of parameter values. Experience has shown that either random or uniform initial estimates of the $\pi$ and $\mathrm{A}$ is adequate for giving useful estimates of these parameters. Equation (2) and Equation (3) show the initial value of $\pi$ and $A$.

$$
\begin{gathered}
\pi_{i 0}=\left\{\begin{array}{l}
0, i \neq 1 \\
1, i=1
\end{array}\right. \\
a_{i j 0}=\left\{\begin{array}{l}
0.5, i=\text { jor } i=j+1 \\
1, i=j=3 \\
0, \text { else }
\end{array}\right.
\end{gathered}
$$

However, for the $B$ parameters, experience has shown that good initial estimates are helpful in CHMM. Based on the sets of the training samples, we applied k-means segmentation with clustering to obtain $B_{0}$. Equation shows description of $B_{0}$.

$$
B_{0}=\left\{b_{j}(o)=\sum_{m=1}^{M} c_{j m} N\left(o, \mu_{j m}, U_{j m}\right)\right\}, 1 \leq j \leq N
$$

Here, $\mathrm{O}$ is the vector being modeled, $C_{j m}$ is the mixture coefficient for the $m$ th mixture in state $\mathrm{j}$ and $\mathrm{N}$ is Gaussian density, with mean vector $\mu_{j m}$ and covariance matrix $U_{j m}$ for the mth mixture component in state $j$. The mixture gains $C_{j m}$ satisfy the stochastic constraint

$$
\begin{aligned}
& \sum_{\mathrm{m}=1}^{M} c_{j m}=1,1 \leq j \leq N \\
& c_{j m} \geq 0,1 \leq j \leq N, 1 \leq m \leq M
\end{aligned}
$$

So that the pdf is properly normalized,

$$
\int_{-\infty}^{\infty} b_{j}(x) d x=1,1 \leq j \leq N
$$

(a) Converting the text files created in step 1, 2, 3 into binary format using MATLAB code. HTK recognizes only binary format for input training files.

(b) Re-estimation the parameters $a_{i j}$ and $b_{j}(O)$ to maximize $P(O \mid \lambda)$. Based on the initial set of parameter values $\lambda_{0}$ and the set of training samples, parameter re-estimation was performed using an embedded training version of the Baum-Welch algorithm.

\section{Results of Driver Intention Recognition}

Intention recognition can be formulated as the evaluation problem for HMM. This problem can be efficiently solved with the Viterbi algorithm, which also embedded in HTK. The task of the trained intention recognition system was to distinguish left and right lane change from normal lane keeping.

The training database for lane change maneuvers consisted of about 400 lane changes, 200 for left and right lane change respectively. The evaluation database consisted of a separate set of various driving sequences with about 200 lane change maneuvers. As is mentioned above, during the test process, for each intention model, three, six and nine states models are evaluated with various window sizes between 1 second to 5 seconds. The results of recognition of LCL intention with different state number and window sizes are reported in Table 2. 
Table 2 Accuracy of recognition of LCL intention with different state number and window sizes

\begin{tabular}{cccc}
\hline \multirow{2}{*}{$\begin{array}{c}\text { Window } \\
\text { time }\end{array}$} & \multicolumn{3}{c}{ State number } \\
\cline { 2 - 4 } & 3 & 6 & 9 \\
\hline 5 & 59.51 & 83.62 & 82.58 \\
3 & 50.31 & 80.23 & 85.34 \\
2 & 49.19 & 89.36 & 90.36 \\
1.5 & 58.63 & 95.48 & 94.56 \\
0.8 & 78.83 & 76.35 & 64.38 \\
\hline
\end{tabular}

As can be read from the confusion matrix in Table 2, the best model for LCL intention recognition was found to be a model of six states of a trained Markov chain with $1.5 \mathrm{~s}$ time window, and the sensitivity of a detection of a left change manoeuvre is $95 \%$. A whole lane change manoeuvre on highway takes on average about six to ten seconds, so the detection is possible rather early in the manoeuvre.

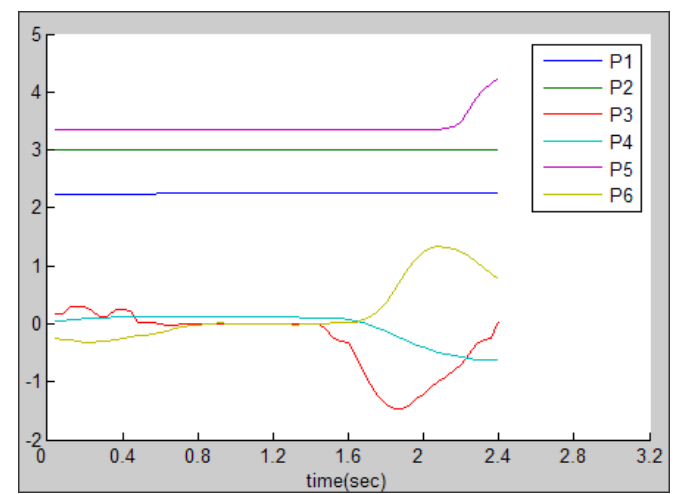

a. LCL manoeuvre at $80 \mathrm{~m} / \mathrm{s}$

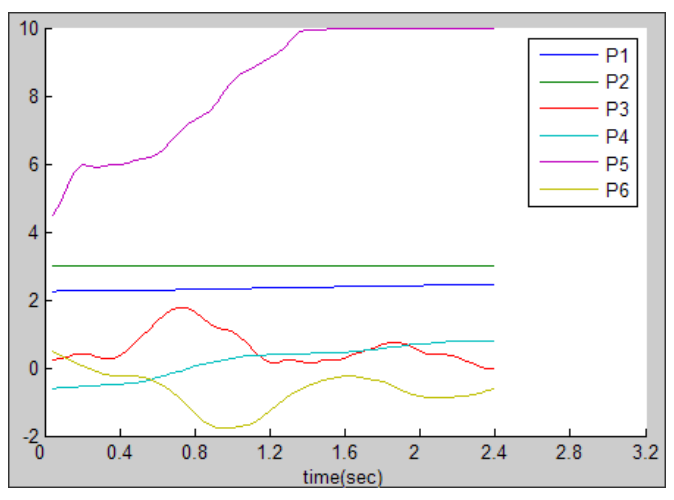

c. LCR manoeuvre at $80 \mathrm{~m} / \mathrm{s}$

\section{Discussions and Conclusion}

A CHMM-based technique for driver intention recognition is development in this paper. The most important factors that affected the results were data representation, size of time window, and initial sets of model parameters. In this paper, different training models were tested to obtain the optimizing model for each driver intention. Experimental results show that, using solely accessible data measured with the driver simulator, such as the steering wheel angle, steering wheel angle velocity and lateral acceleration can achieve ideal accuracy, and it proved very effective in terms of early intention recognition.

It is also likely that calculating new features from other signals would be helpful. Before changing lane, driver gaze orientation shows certain regularity, e.g. 'look left-look ahead-look left-look ahead-...' So the acquisition of driver gaze orientation and its sequences may effectively increase the recognition efficiency.

Also, since the framework is generic, other intention models like turn and park intention can also be developed based on this framework.

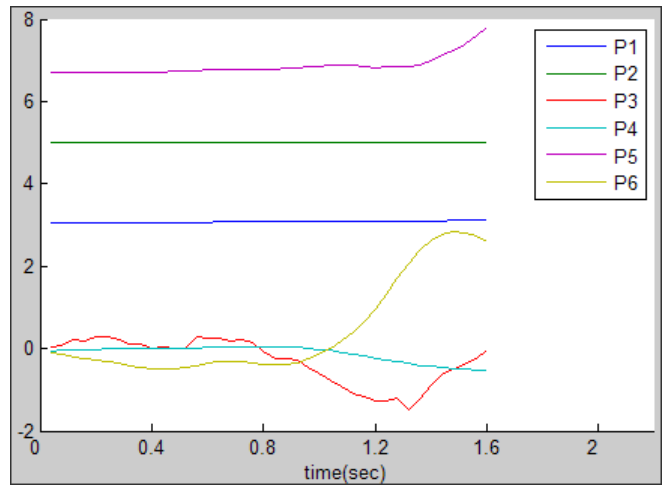

b. LCL manoeuvre at $110 \mathrm{~m} / \mathrm{s}$

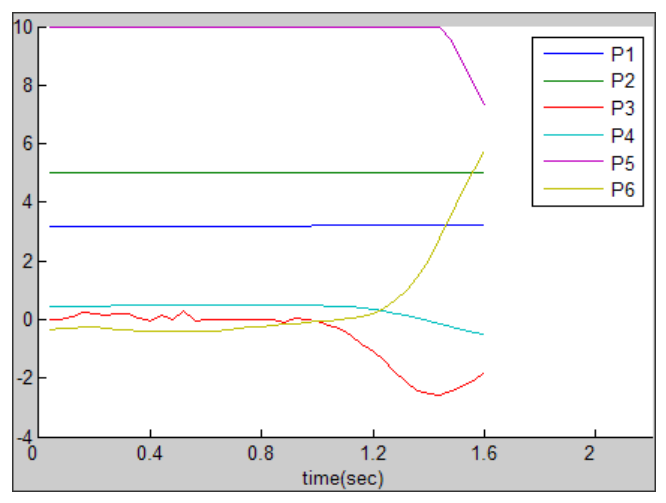

d. LCR manoeuvre at $110 \mathrm{~m} / \mathrm{s}$ 


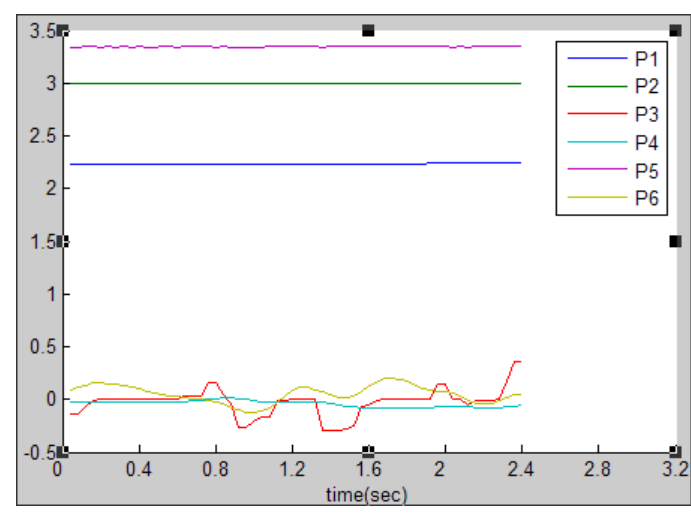

e. LCR manoeuvre at $80 \mathrm{~m} / \mathrm{s}$

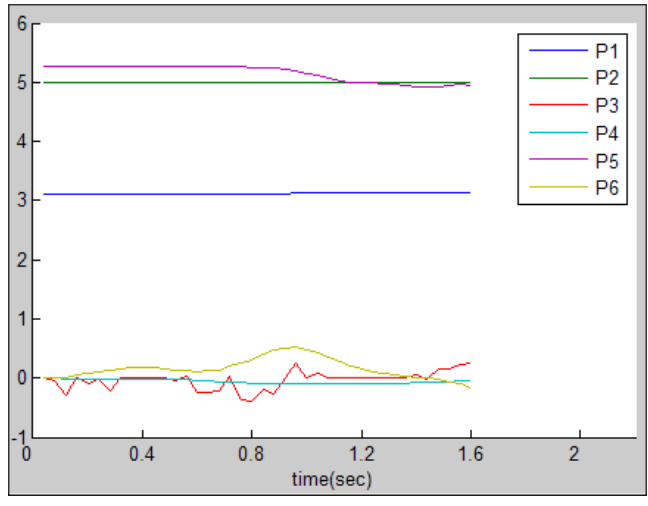

f. LCR manoeuvre at $110 \mathrm{~m} / \mathrm{s}$

Fig. 2. Examples of parameters during a lane change manoeuvre

\section{Acknowledgements}

This research was supported partly by National Nature Science Foundation of China (No.50908098), Program for New Century Excellent Talents in University, and the Basic Research Project of Jilin Provincial Science and Technology Department (No.20100516).

\section{References}

1. Interim Report 2000, The TEA-21 ITS Deployment Program, U. S. Department of Transportation, www.its.dot.gov.

2. European transport policy for 2010: time to decide, White paper, European transport policy , ISBN 92-894-0341-1.

3. W. H. Wang, F. G. Huo, H. C. Tan and H. Bubb, A framework for function allocation in intelligent driver interface design for comfort and safety, International Journal of Computational Intelligence Systems, 3(5)(2010), pp. 531-541.

4. G. Novak, A. Bais, and M. Stefan. Simple stereo vision system for real-time object recognition for an autonomous mobile robot. Second IEEE International Conference on Computational Cybernetics, 2004, pp 213-216.

5. C. Wahlgren, T. Duckett, Topological map building for mobile robots using omnidirectional vision, The Third Swedish Workshop on Autonomous Robotics (SWAR05), Kista, Stockholm, 2005.

6. B. Steux, L. Salesse, D. Wautier, Fade: a vehicle detection and tracking system featuring monocular color vision and data fusion. IEEE Symposium on Intelligent Vehicles, France, 2002, pp 845-846.
7. A. Broggi, S. Cattani, P. P. Porta, et al.. A laserscanner-vision fusion system implemented on the TerraMax autonomous vehicle. Proceedings of the 2006 IEEE/RSI International conference on intelligent robots and systems, Beijing, 2006, pp 111-116.

8. I. Bauermann and E. Steinbach. Joint calibration of a range and visual sensor for the acquisition of RGBZ concentric mosaics. VMV2005, Germany.

9. W. Barfield and T. A. Dingus, Human factors in intelligent transportation systems (Lawrence Erlbaum Associates, Inc. New Jersey, 1997).

10. R. Sukthankar, J. Hancock, C. Thorpe, Tactical-level Simulation for Intelligent Transportation Systems, Mathematical and Computer Modelling, 27(1998), 19-24.

11. E.C.B.Olson, Modeling slow lead vehicle lane changing, dissertation, Virginia Polytechnic Institute and State University, Blacksburg VA, 2003.

12. G. S. Aoude and J. P. How, Using Support Vector Machines and Bayesian filtering for classifying agent intentions at road intersections, Massachusetts Institute of Technology, Tech. Rep. ACL09-02, 2009.

13. C. A. Pribe, S. O. Rogers, Learning to associate observed driver behavior with traffic controls. Transportation Research Record: Journal of the Transportation Research Board, Vol. 1679/1999. pp. 95-100.

14. J. C. McCall and M. M. Trivedi, Human behavior based predictive brake assistance. IEEE Intelligent Vehicles Symposium, 2006, pp. 8-12.

15. D. D. Salvucci, Inferring driver intent: a case study in lane-change detection, proceedings of the human factors ergonomics society 48th annual meeting, 2004.

16. L. R. Rabiner and B. H. Juang, An introduction to Hidden Markov Models, IEEE ASSP MAGAZINE, 1986, Vol.3, pp.4-16.

17. A.Pentland, A.Liu, Modeling and prediction of human behavior. Neural Computation 1999. Vol.11, pp. 229-242. 
18. T.Mizushima, P. Raksincharoensak and M. Nagai. Direct yaw-moment control adapted to driver behavior recognition. SICE-ICASE International Joint Conference. 2006, pp. 534-539.

19. D. Meyer-Delius, C.Plagemann, W.Burgard, Probabilistic situation recognition for vehicular traffic scenarios. Proceedings of the 2009 IEEE international conference on Robotics and Automation, pp. 4161-4166.
20. L.Rabiner, A tutorial on hidden markov models and selected applications in speech recognition. In Proceedings of the IEEE, vol.77 (2), 1989, pp.257-286.

21. S.Young, The HTK hidden markov model toolkit: Design and philosophy, Tech. Rep. TR153, Department of Engineering, Cambridge University, UK, 1993.

22. HTK Speech Recognition Toolkit, http://htk.eng.cam.ac.uk. 